\title{
Adaptive sequence evolution in a color gene involved in the formation of the characteristic egg-dummies of male haplochromine cichlid fishes Walter Salzburger ${ }^{\dagger 1,2,3}$, Ingo Braasch ${ }^{\dagger 1,4}$ and Axel Meyer*1
}

Address: ${ }^{1}$ Lehrstuhl für Zoologie und Evolutionsbiologie, Department of Biology, University Konstanz, 78457 Konstanz, Germany, ${ }^{2}$ Department of Ecology and Evolution, University of Lausanne, 1015 Lausanne, Switzerland, 3Zoologisches Institut der Universität Basel, Evolutionsbiologie, Universität Basel, 4051 Basel, Switzerland and 4Physiological Chemistry I, University of Würzburg, 97074 Würzburg, Germany

Email: Walter Salzburger - walter.salzburger@unibas.ch; Ingo Braasch - ingo.braasch@biozentrum.uni-wuerzburg.de; Axel Meyer* - axel.meyer@uni-konstanz.de

* Corresponding author †Equal contributors

Published: 15 November 2007

BMC Biology 2007, 5:5। doi:|0.|I86/|74|-7007-5-5।
Received: 20 September 2007

Accepted: 15 November 2007

This article is available from: http://www.biomedcentral.com/174I-7007/5/5I

(c) 2007 Salzburger et al; licensee BioMed Central Ltd.

This is an Open Access article distributed under the terms of the Creative Commons Attribution License (http://creativecommons.org/licenses/by/2.0), which permits unrestricted use, distribution, and reproduction in any medium, provided the original work is properly cited.

\begin{abstract}
Background: The exceptionally diverse species flocks of cichlid fishes in East Africa are prime examples of parallel adaptive radiations. About $80 \%$ of East Africa's more than I 800 endemic cichlid species, and all species of the flocks of Lakes Victoria and Malawi, belong to a particularly rapidly evolving lineage, the haplochromines. One characteristic feature of the haplochromines is their possession of egg-dummies on the males' anal fins. These egg-spots mimic real eggs and play an important role in the mating system of these maternal mouthbrooding fish.
\end{abstract}

Results: Here, we show that the egg-spots of haplochromines are made up of yellow pigment cells, xanthophores, and that a gene coding for a type III receptor tyrosine kinase, colony-stimulating factor I receptor a (cs $/ \mathrm{ra}$ ), is expressed in egg-spot tissue. Molecular evolutionary analyses reveal that the extracellular ligand-binding and receptor-interacting domain of csflra underwent adaptive sequence evolution in the ancestral lineage of the haplochromines, coinciding with the emergence of egg-dummies. We also find that csflra is expressed in the egg-dummies of a distantly related cichlid species, the ectodine cichlid Ophthalmotilapia ventralis, in which markings with similar functions evolved on the pelvic fin in convergence to those of the haplochromines.

Conclusion: We conclude that modifications of existing signal transduction mechanisms might have evolved in the haplochromine lineage in association with the origination of anal fin eggdummies. That positive selection has acted during the evolution of a color gene that seems to be involved in the morphogenesis of a sexually selected trait, the egg-dummies, highlights the importance of further investigations of the comparative genomic basis of the phenotypic diversification of cichlid fishes.

\section{Background}

Cichlid fishes in general, and the species flocks of cichlids in the East African Great Lakes in particular, are premier examples of animal adaptive radiations [1-4]. The cichlid species flocks of Lakes Victoria, Malawi and Tanganyika are an excellent model system to study the genetic basis of organismal diversity, because of their enormous phenotypic diversity, their species-richness, their close related- 
ness and the repeated occurrences of evolutionary parallelisms (see e.g., $[2,3,5,6]$ ). About $80 \%$ of East Africa's endemic cichlid species, and all species of the species flocks of Lakes Victoria and Malawi (plus one lineage from Lake Tanganyika) belong to a particularly successful lineage of cichlids, the haplochromines [7-9]. Only a few synapomorphies of the haplochromines have been identified to date. All taxa appear to have a similar type of upper pharyngeal bones [10]. Two additional characteristic features of the haplochromines are a polygynous and/ or polygynandrous mating system and maternal mouthbrooding, with only females incubating the eggs in their buccal cavities, as well as 'egg-dummies' (ocelli) in the form of ovoid markings on the anal fins of males that mimic real eggs in size, color and shape [7,11-16] (Figure 1).

The males' yellow to orange egg-spots play a crucial role in the haplochromines' mating system in that they serve as intra-specific sexual advertisement to attract females and to maximize breeding success $[11,16,17]$, possibly as a response to predation pressure from con-specifics [18]: A female with ripe eggs initiates breeding by approaching a territorial courting male, which responds with a lateral display in the form of quivering and fluttering the unpaired anal, caudal and dorsal fins, thereby exposing its bright coloration. The female lays a small batch of eggs (Figure 1b), circles around, and picks them up in her mouth (Figure 1c) before fertilization. Attracted by the egg-spots on the males' anal fin, the female attempts to ingest these dummies and brings her mouth into close proximity to the males' genital opening - this is when the male discharges sperm (Figure 1d; see Additional file 1). In this way, the fertilization of the eggs takes place within the females' mouth $[11,12,14,15]$. We note that some variations from this general procedure have been reported. For example, in some species the eggs are fertilized before being picked up by the female [19]. Haplochromine females were shown to prefer males with egg-spots over males where the dummies had been removed [17] or manipulated experimentally [20], and it has been suggested that sexual selection on egg-spot morphology could lead to speciation [21].

Haplochromine egg-dummies begin to form in the juvenile stage of males and start out from the edge of the anal fin [12], though they only begin to brighten when the young males reach sexual maturity. Haplochromine species differ greatly in egg-spot number, arrangement and morphology. Anal fin egg-dummies are, however, not an exclusive male characteristic. In some species, females also show ovoid markings on their anal fins, but these are typically much less conspicuous than the egg-spots of males. A typical haplochromine egg-dummy is characterized by a conspicuous yellow to reddish central area and a more or less transparent outer ring $[11,12,14]$. This type of eggdummy is found in most riverine and rock-dwelling haplochromines, while other species, such as more ancestral riverine taxa or pelagic and sand-dwelling species in Lake Malawi, sometimes show a more amorphic blotch pattern. Additionally, a small number of extant haplochromines lack egg-spots entirely, and it has been suggested that they have lost their dummies secondarily [7].

Yellow blotches that also act as egg-dummies are also known from a few species of the Lake Tanganyika tribe Ectodini, which are only distantly related to the haplochromines (see e.g., [22]). However, the ectodines' eggdummies are morphologically less complex than those of the haplochromines and they are found on the paired pelvic fins instead of the unpaired anal fins as in haplochromines. Finally, some mouthbrooding Tilapia species have evolved filamentous arborescent appendages at their genital papillae, so-called genital tassels, which act as eggdummies $[11,14]$.

Here, we report on the identification of a gene that is likely to play a role in the development of the yellow xanthophores in the haplochromines' egg-spots. We hypothesized that a previously isolated xanthophore-related color gene might be involved in the formation of xanthophores in the egg-spots: The colony-stimulating factor 1 receptor a (csf1 ra) gene, coding for a type III receptor tyrosine kinase $[23,24]$, is known to be expressed by cells of the xanthophore lineage in zebrafish; it is essential for recruiting xanthophores from their precursors, and it is indirectly involved in the organization of the dark melanophores $[25,26]$. Zebrafish mutants for csf1ra (panther) exhibit disrupted stripe patterns and lack xanthophores [25], and it has been shown that some species in the genus Danio vary in their csf1ra pathway during pigment pattern formation [27]. csfira is, thus, not only an important marker for the xanthophore lineage in zebrafish, but is also involved in xanthophore development and, possibly, color patterning. In addition to its role in body coloration, csf1ra is expressed in the macrophage and osteoclast cell lineage in zebrafish [25]. We performed RT-PCR and in situ hybridization experiments in several haplochromine cichlid species in order to confirm the expression of csfira in the males' egg-spots. For detailed evolutionary analyses, we determined the DNA sequence of this gene locus in 19 East African cichlid species and tested for the signature of adaptive evolution in the haplochromine lineage. Finally, we also tested for csf $1 \mathrm{ra}$ expression in the ectodine species Ophthalmotilapia ventralis, which has egg-dummies at the end of the paired pelvic fins. 


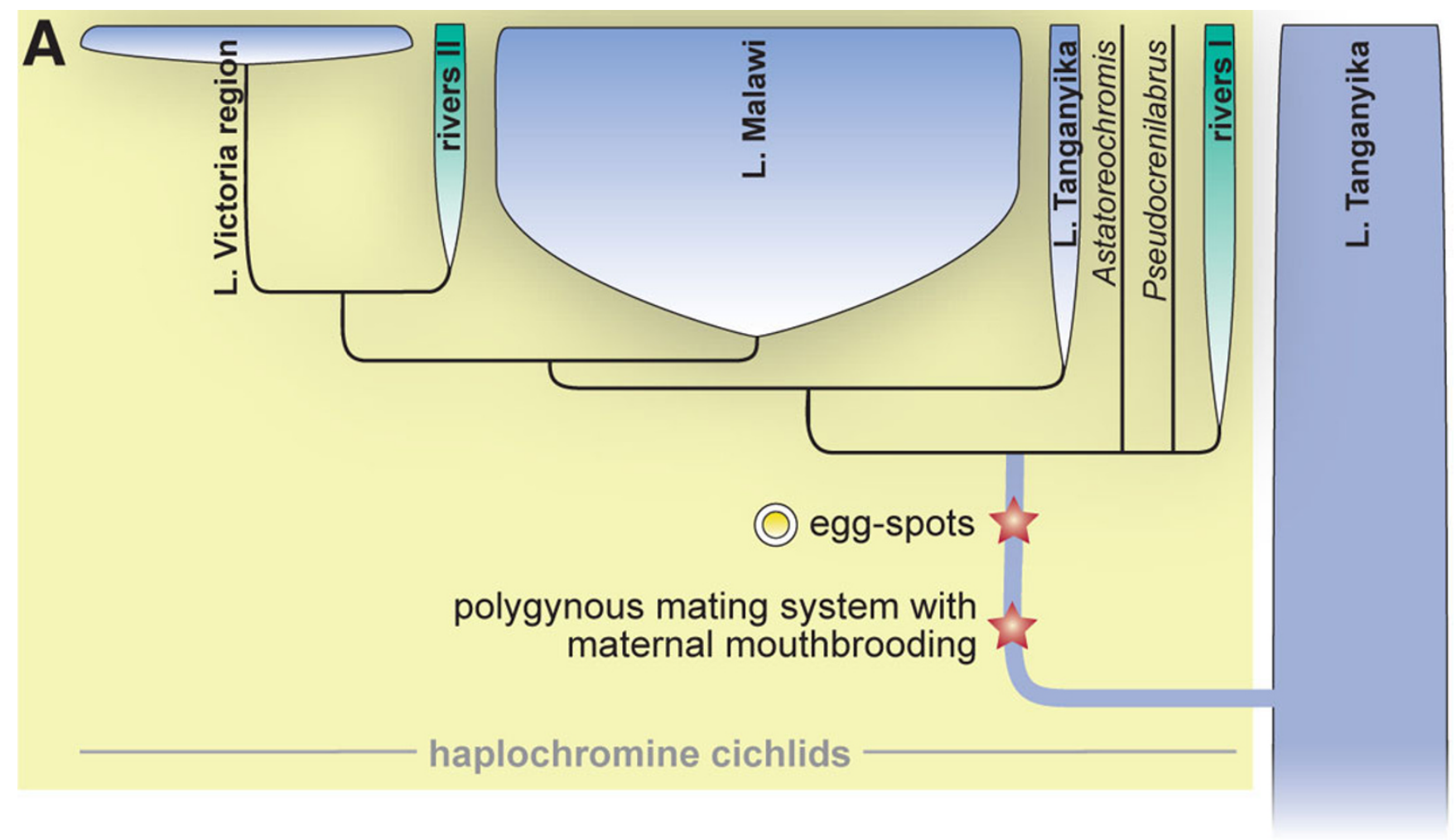

B

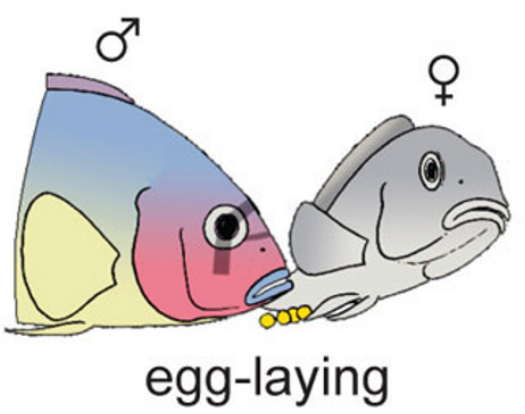

C

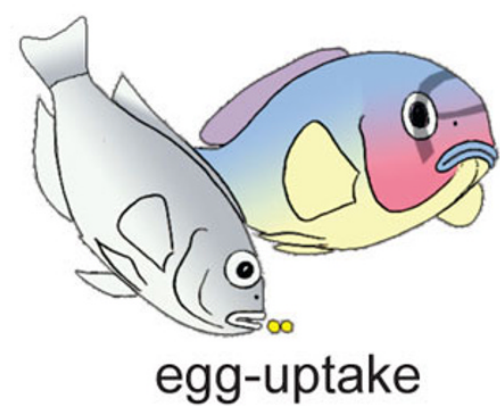

D

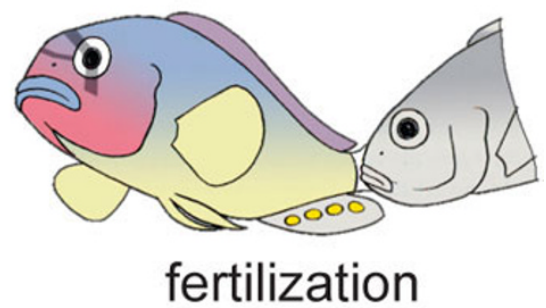

Figure I

Haplochromine evolution in Africa. (a) Consensus phylogeny of East African cichlids based on mitochondrial [7] and nuclear DNA markers (this study). The haplochromines are derived from the much older cichlid species assemblage of Lake Tanganyika. In only about two million years of evolution, the haplochromines gave rise to spectacular adaptive radiations in Lakes Tanganyika ( 30 species), Malawi (up to 1000 species) and Victoria ( 500 species), as well as in other lakes and rivers in Africa (up to 200 species) [1,3,7-9]. Evolutionary innovations are mapped onto the phylogeny [7]. Note that mouthbrooding evolved several times during cichlid evolution, but only the haplochromines show a characteristic polygynous maternal mouthbrooding system with egg-spots as sexual advertisement [7,II-I4,I7]. (b-d) The egg-spots on the anal fins of male haplochromines play a crucial role in the haplochromines' reproductive system. Three main phases of the spawning cycle are shown: (b) the female lays a small batch of eggs in the territory of a male. (c) The female takes up the eggs into her mouth. (d) The male presents its conspicuous egg-spots on his anal fin. The female is attracted by these egg-dummies and tries to pick them up, thereby bringing her mouth close to the male's genital opening, through which sperm is released.

\section{Results}

\section{Gene expression assays}

By applying a fluorescence-based detection method, we first confirmed that the yellowish center of the haplo- chromines' egg-spots consists of a particular class of pigment cells, the xanthophores (Figure 2). The subsequent RT-PCR experiments from egg-spot tissue of A. burtoni were positive for the xanthophore-related color gene 


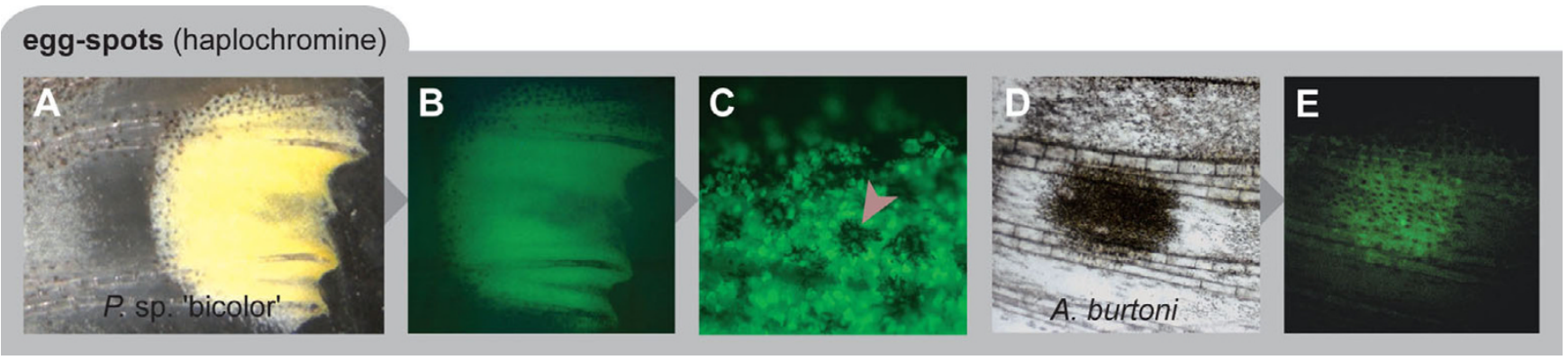

\section{Figure 2}

The haplochromines' egg-spots are made up of xanthophores. (a-c) Photograph (a) and fluorescence image (b, c) of the egg-spot of Pseudotropheus sp 'bicolor'. The yellow inner ring of the egg-spot of $P$. sp. 'bicolor' consists of xanthophores, the pteridine pigments of which fluoresce at high $\mathrm{pH}$. Melanophores are also present in the border area around the transparent outer ring (a melanophore is marked by an arrowhead). (d, e) Egg-spot of Astatotilapia burtoni; bright field (d) and fluorescence image (e).

csf1ra. This was further confirmed by in situ hybridization experiments documenting csfira expression in the males' egg-dummies in all tested haplochromine species that displayed these ovoid markings (Figure 3a-k). In Astatotilapia burtoni males, which have up to a dozen egg-spots that are organized in two or more rows, we detected csf1 ra expression in the developmentally younger and still growing egg-spots in proximity to the edge of the anal fin (Figure $3 a-d)$, which is exactly where new egg-dummies begin to form [12]. Males of the Pseudotropheus sp. 'bicolor' population used for this study show a single egg-spot only, in which $c s f 1 r a$ is also expressed (Figure $3 \mathrm{e}-\mathrm{h}$ ). Similarly, csfira expression was detected in the yellow markings of Thoracochromis brauschi (Figure 3i-k), a member of a basal riverine haplochromine lineage [7] with rather unstructured yellow blotches. The egg-spot free anal fins of $P$. multicolor $[7,12]$, however, do not show csfira expression (Figure $3 \mathrm{~m}-\mathrm{p}$ ). This is not due to the use of a heterologous probe designed on the basis of csf1ra from A. burtoni, as other patterns in P. multicolor skin revealed csf1 ra positive results (see below). Female anal fins and negative controls did not show any labeling (Figure 3). Furthermore, we determined csf1 ra expression in the pearly spot pattern in the dorsal fin of A. burtoni and P. multicolor (Figure 3q-u) and in the posterior part of the anal fin of T. brauschi (Figure 3l). Expression of csf1 ra was also detected in the tassels at the tips of the conspicuously elongated paired ventral fins of males of the non-haplochromine Tanganyikan species Ophthalmotilapia ventralis, where these yellow markings also function as egg-dummies [14] (Figure 3v-x), but not in the similarly elongated female ventral fins of $O$. ventralis that lack egg-dummies (Figure $3 \mathrm{y}, \mathrm{z}$ ).

\section{Phylogenetic and molecular evolutionary analyses of the csfl ra locus}

We sequenced more than 7000 bp of the csf1ra locus of 19 cichlid species representing the phylogenetic diversity of cichlids in East Africa (Figure 4a-c). Our phylogenetic analyses, based on the intron sequences of the csf1 ra locus (>4 000 bp; Figure 4d), confirmed previous phylogenetic hypotheses by placing the representatives of the Bathybatini and Trematocarini as sister group to a clade comprised by the substrate spawning lamprologines (represented by A. calvus, L. teugelsi, and T. bifrenatus) and the East African mouthbrooding cichlids (remaining taxa) $[7,22,28]$. The haplochromines (yellow box in Figure $4 d$ ) form a strongly supported monophyletic clade within these mouthbrooders, with P. multicolor, T. brauschi and A. alluaudi branching off ancestrally to the exceptionally species-rich 'modern haplochromines' represented by A. burtoni, C. moorii, H. bloyeti, H. obliquidens, $P$. sp., and $X$. phytophagus [7]. The bootstrap values were generally high, except for the branches connecting the four main lineages of haplochromines. Just as in our previous molecular phylogenetic analysis [7], the Shimodaira-Hasegawa test did not favor a particular phylogenetic hypothesis with respect to the interrelationship of the four main lineages of haplochromines.

The ancestral state reconstructions using a consensus tree on the basis of this newly-generated phylogenetic hypothesis (Figure $4 \mathrm{~d}$ ) and a previously published mitochondrial phylogeny [7] revealed that both the particular mating system and the egg-dummies on male anal fins are likely to have evolved only once in the ancestral lineage of the haplochromines (see Figure 1a). Thus, the lack of eggspots in a few haplochromine species is most likely due to secondary loss.

We then tested for the existence of a signal of adaptive sequence evolution in the coding region of csf1ra. This gene consists of 21 exons with a combined length of 2928 $\mathrm{bp}$, and is made up of a cysteine-rich extracellular ligandbinding domain composed of five immunoglobulin-like 

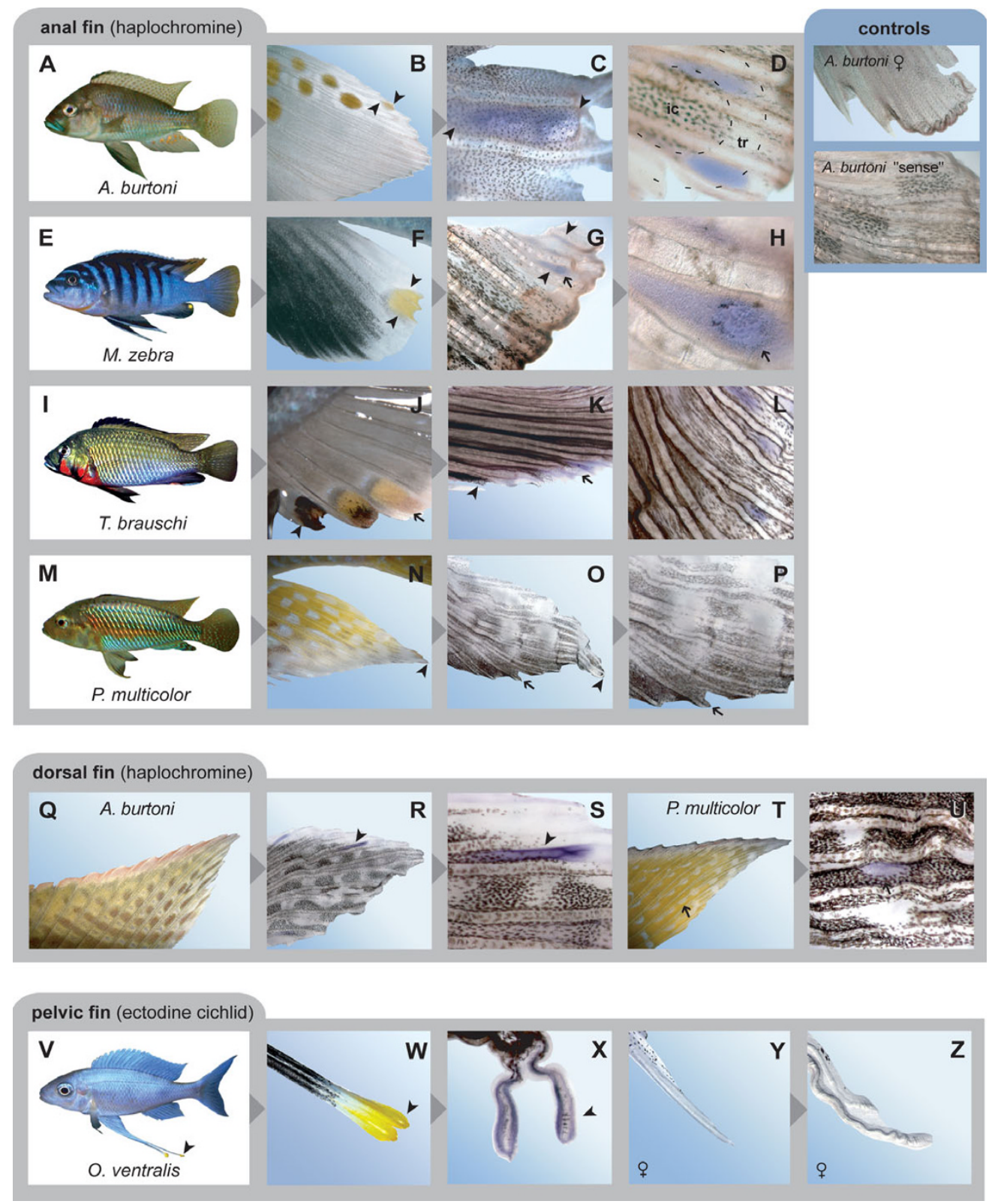

\section{Figure 3}

In situ hybridization experiments showing csfI ra expression in cichlids. (a-d) In male Astatotilapia burtoni (a), which typically have more than 10 egg-spots arranged into two or more rows (b), we could detect csfl ra expression in the forming (c) as well as in the growing egg-dummies (d; the position of the yellowish inner circle, ic, and the transparent outer ring, tr, is indicated). Note that the yellow pigments of the egg-spots are removed during tissue processing. (e-h) In male Pseudotropheus sp. 'bicolor' (e), for which we used a morph with only one egg-spot (f), csfl ra expression was detected throughout the entire egg-spot (g), and it appears that the dark melanophores arrange around the center of csfl ra expression (h), just as reported for zebrafish, where csfl ra is indirectly also responsible for melanophore organization [25]. (i-I) In male Thoracochromis brauschi (i), csfl ra is expressed in the yellow blotches of the anal fin (j, k), and also in the pearly spots ('Perlfleckmuster') on the posterior part of the anal fin $(\mathrm{l}) .(\mathrm{m}-\mathrm{p})$ In the basal riverine haplochromine Pseudocrenilabrus multicolor $(\mathrm{m})$, the male anal fins do not exhibit egg-spot like blotches (n) and no csfl ra expression could be detected either (o, p). ( $q-u) c s f l r a$ is expressed in the yellowish/orange areas of the pearly spot pattern on the dorsal fins of male A. burtoni $(\mathrm{q}-\mathrm{s})$ and $P$. multicolor $(\mathrm{t}, \mathrm{u})$. ( $\mathrm{v}-\mathrm{z}) \mathrm{csf} / \mathrm{ra}$ is expressed in the egg-dummies on the tassels at the tips of the conspicuously elongated pelvic fins of the ectodine cichlid Ophthalmotilapia ventralis $(v-x)$. Female fins of $O$. ventralis $(y)$ do not show csfl ra expression $(z)$. The control experiments show that csfl ra is not expressed in female anal fins or in the "sense" control. Arrowheads refer to identical points in sequential images. 
A

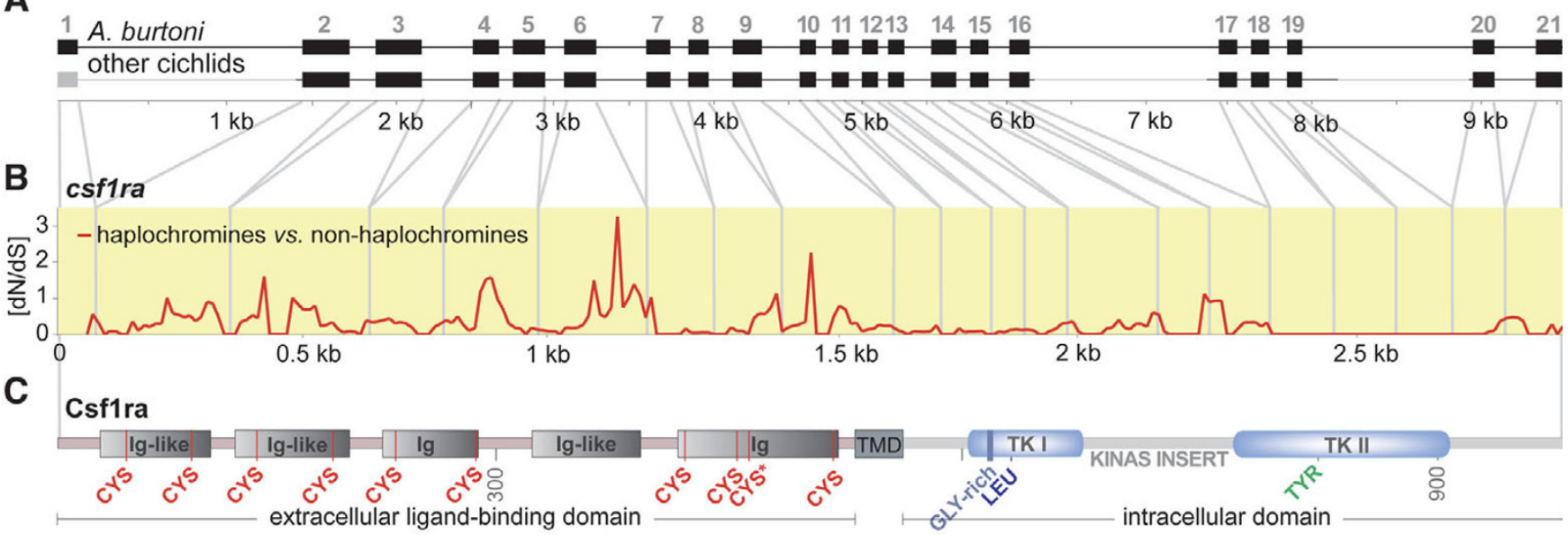

D

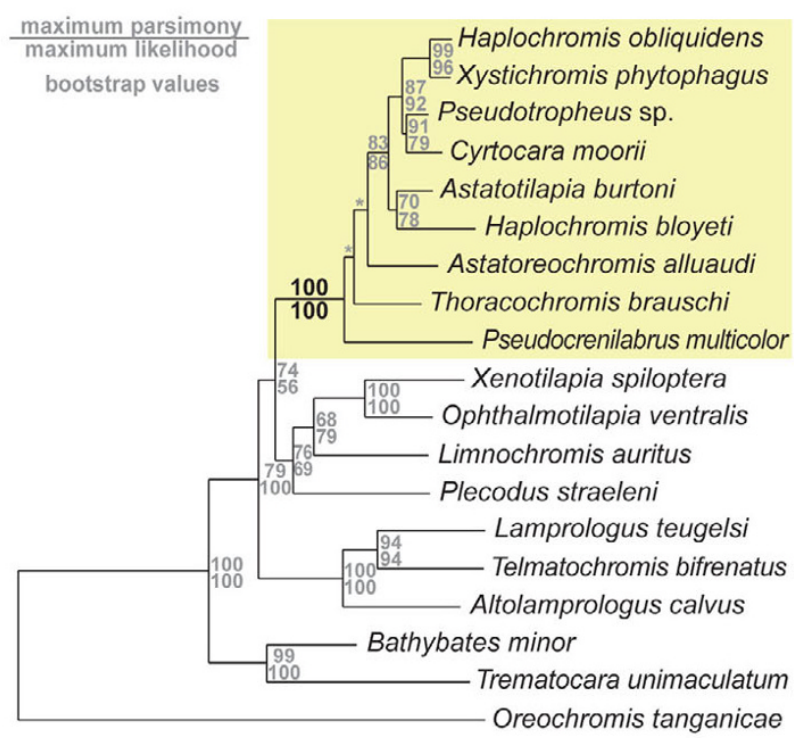

0.005 substitutions/site

\section{E}

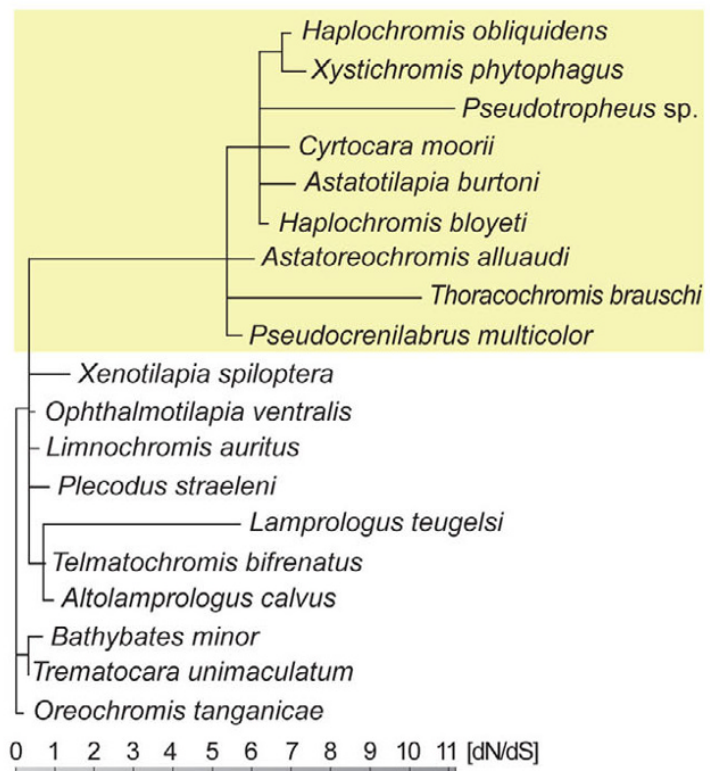

Figure 4

Molecular evolutionary analyses of the csf Ira locus. (a) The entire csf I ra gene locus was obtained from 19 cichlid species (exon I and the relatively large introns I, 16, and 19, which are shown in gray, were not sequenced). (b) dN/dS ratio of haplochromines compared to non-haplochromine cichlids as revealed from a sliding window analysis with DNASP. (c) Schematic representation of the structure of the Csf I ra protein. The gene consists of an extracellular ligand-binding domain containing five immunoglobulin-like (lg-like) domains with conserved cysteines (CYS), a transmembrane domain (TMD), and an intracellular partition that contains two tyrosine kinase (TK) domains interrupted by a kinase insert domain. In the tyrosine kinase I domain (TK I) a glycine-rich region (GLY-rich) and a conserved leucine (LEU) is found, the tyrosine kinase II domain (TK II) contains a conserved tyrosine (TYR). The asterisk indicates that in one species, $X$. phytophagus, the otherwise conserved cysteine at amino acid position 450 has been replaced by a tryptophan. (d) Maximum likelihood phylogeny based on more than 4100 bp of non-coding sequences of the csfl ra gene locus corroborating the monophyly of the haplochromines (yellow box) and the ancestral position of A. alluaudi, P. multicolor, and T. brauschi [7]. The asterisk indicates relatively short branches not supported by high bootstrap values or a Shimodaira-Hasegawa test. (e) Branch-scaled tree showing the $d N / d S$ rates reconstructed with HyPhy. The only internal branch with a $\mathrm{dN} / \mathrm{dS}>\mathrm{I}$ is the one representing the common ancestor of the haplochromines (yellow box). 
domains, a transmembrane domain, and an intracellular domain with two separate tyrosine kinase domains $[23,29]$ (Figure $4 a, c)$. A sliding window analysis with DNASP [30] uncovered several sections in csf1ra of haplochromines with a $d \mathrm{~N} / d \mathrm{~S}$ ratio greater than one (Figure $4 \mathrm{~b})$, which would indicate that positive selection has acted to shape the protein. These regions were primarily located in the first $1551 \mathrm{bp}$ of the gene (amino acid positions 1 to 517) corresponding to the extracellular ligandbinding domain of Csf1ra. In this domain, there are in total more non-synonymous substitutions than synonymous ones in the haplochromines (see amino acid alignment in Additional file 2), but not in the more basal cichlid lineages that are without anal fin egg-dummies. The maximum likelihood reconstructions of $d \mathrm{~N} / d \mathrm{~S}$ ratios in this domain of csf1ra revealed that the only internal branch with a $d \mathrm{~N} / d \mathrm{~S}>>1$ is the one representing the common ancestor of the haplochromines $(d \mathrm{~N} / d \mathrm{~S}>5$; Figure $4 \mathrm{e}$ ). Such a signal of adaptive sequence evolution specific to the haplochromine lineage could not be detected in a segment of the extracellular domain of kita, another type III receptor tyrosine kinase with a known function in pigment patterning [31], which we have sequenced in the 19 cichlid species as a control. Also, in kita there was no indication that the segment of the extracellular domain would have evolved under a different selection regime as compared to a segment in the intracellular domain (Additional file 3 ).

We also analyzed a genomic region of approximately 1 000 bp upstream of csf1ra in the 19 cichlid species, to investigate sequence differences in putative gene regulatory regions. The only mutation common to all haplochromines, distinguishing them from the more ancestral and less species-rich cichlid lineages, involves a mutation in a putative transcription factor-binding site, a TATA box, about 130 bp upstream of csfira.

\section{Discussion \\ csf I ra is expressed in egg-dummies of haplochromine cichlids}

Egg-dummies on the male anal fins play an important role during the breeding behavior of the female mouthbrooding haplochromines (see e.g., $[7,13,14])$. Phylogenetic and character state reconstructions corroborate that these egg-dummies evolved only once in the ancestral lineage of haplochromines [7] (Figure 1a), and that the absence of ovoid markings on the anal fins of some haplochromine species is due to secondary loss, e.g. because of reasons of camouflage or as an adaptation to the deep-water habitat and visual environment where these markings would not be easily visible.

Haplochromine egg-spots vary in size, shape, number and arrangement in different species. Figure 3 provides some examples of the diversity of egg-spots found in haplochromines. A typical egg-dummy consists of a conspicuous yellow to reddish central area and a more or less transparent outer ring $[11,12,14]$ (see Figure $3 b, f$ ), although a number of species only show amorphic blotches (see e.g., Figure 3j). The brightly colored inner circle is, as we have shown (Figure 2), made up of xanthophores. Yellowish, orange, or reddish xanthophores also occur in cichlid fins other than the anal fin (see e.g., Figure $3 q-u$ ) and in skin tissue, where substantial differences in densities - depending on coloration patterns - can be found (Clabaut, Salzburger and Meyer, unpublished results). However, nowhere (not even in yellow colored fish) could we identify a higher density of xanthophores than in the egg-dummies of haplochromine males.

We applied a candidate gene approach in order to test whether the previously isolated xanthophore-related color gene csfira $[25,26]$ is expressed in haplochromine egg-spots. Our in situ hybridization experiments indeed corroborate csf1 ra expression in the egg-dummies of all tested haplochromine species (Figure 3 ). We detected csfira expression in the younger and still growing eggspots of A. burtoni (Figure 3a-d), in the single egg-dummy of P. sp. 'bicolor' (Figure $3 \mathrm{e}-\mathrm{h}$ ), and in the relatively unstructured male anal fin blotches of $T$. brauschi (Figure $3 \mathrm{i}-\mathrm{k}$ ), which is a member of an ancestral riverine haplochromine clade. The simple orange blotches of $T$. brauschi (Figure $3 \mathrm{j}$ ) again illustrate (see above) that not all types of egg-dummies show a clear-cut separation into a brightly colored inner circle and a more or less transparent outer ring. In this specific case, it could, however, be argued that because of the phylogenetic position of this species, the undifferentiated spots of $T$. brauschi represent an intermediate character state in the evolution of egg-dummies. That csf1 ra is expressed in egg-spots of younger males and the smaller and still developing egg-spots of adult males might indicate that csf1ra is required for xanthophore recruitment from pigment cell precursors during egg-spot formation, just as has been reported for the formation of stripes in zebrafish $[25,26]$. This would need to be tested in future experiments.

In view of the fact that csf1 $\mathrm{ra}$ is expressed in both patterns, the comparative in situ hybridization experiments also seem to support the earlier suggestion that egg-spots of haplochromines are likely to be derived from the 'PerIfleckmuster' found in unpaired fins of haplochromines and many other cichlid species $[11,12]$. Specifically, we show that csf1 ra is expressed in pearly spots on dorsal fins of A. burtoni and P. multicolor (Figure 3q-u). As such pearly spots are also found on anal fins of some (ancestral) haplochromine lineages (see Figure 3l for csfira expression in the pearly spots on the posterior part of the anal fin of T. brauschi), a co-option of at least some aspects 
of the molecular basis of the pearly blotch pattern for the formation of egg-dummies appears likely.

\section{Adaptive sequence evolution in the extracellular domain of csf I ra in haplochromines}

In order to investigate the molecular evolutionary history of csf1ra we sequenced large fractions of the locus in 19 representative cichlid species (Figure 4a). The comparison of haplochromine with non-haplochromine species revealed that several regions in csf1ra show a $d \mathrm{~N} / d \mathrm{~S}$ ratio greater than one (Figure $4 \mathrm{~b}$ ), indicating that positive selection (adaptive sequence evolution) has acted to change the protein in haplochromines. Positive Darwinian selection often only acts on particular domains of a gene, whereas other sections remain subject to purifying selection. Also, adaptive evolution is expected to act only at particular times during the evolution of a lineage. In our case, we found that the regions showing a $d \mathrm{~N} / d \mathrm{~S}>1$ are located in the part of the gene that encodes the extracellular domain. The Csf1ra protein functions as membrane spanning cell surface receptor $[29,32]$, and is characterized by a cysteine-rich extracellular ligand-binding domain composed of five immunoglobulin-like domains containing growth factor binding sites, a transmembrane domain, and two separate tyrosine kinase domains $[23,29]$ (Figure 4c). A maximum likelihood reconstruction of $d \mathrm{~N} / d \mathrm{~S}$ ratios revealed that the extracellular domain of csf1 ra underwent adaptive evolution in the common ancestor of the haplochromines (Figure 4e) - simultaneous to when the egg-dummies are likely to have evolved. The occurrence of adaptive changes in the amino acid sequence in the ligand-binding portion of csf1ra (Figure $4 e$, Additional file 2) seems to suggest that novel modifications of existing signal transduction mechanisms evolved in the haplochromine lineage that were associated with the evolution of egg-spots, or, possibly, other color patterns involving xanthophores. Functional assays (see e.g., [33]) are now required to test whether the observed differences in the coding sequence of csf1 ra have any effect on ligand-receptor interactions. Similarly, a more thorough comparative analysis of the upstream region of $c s f 1 r a$ is necessary to test the possibility that regulatory elements also underwent evolutionary changes in the ancestor of haplochromines, as is suggested by the observed haplochromine-specific mutation in a putative transcription factor binding site and the differential expression of csf1ra between haplochromine and nonhaplochromine cichlids. In addition, csf1ra expression should also be investigated in other tissues and cell lineages. Although it is not apparent how e.g., macrophages or osteoclasts (in which csf1ra is also expressed [25]) could contribute to the evolutionary success of haplochromines but not of other cichlids, the remote possibility remains that the signal of adaptive sequence evolution is due to functions other than coloration.
To date, evidence for accelerated protein evolution in haplochromines has been found in the bone morphogenetic protein 4 (bmp4) that is hypothesized to be involved in jaw formation ([34]; see also [5,35]), in a color perception gene, the long wavelength-sensitive (LWS) opsin [36], and in a putative color gene, the F-box-WD-repeat hagoromo [37]. The finding of signatures of adaptive sequence evolution in a jaw-related gene, as well as in color and colorperception genes, seems to corroborate the hypothesis that both the particular architecture of the cichlids' jaw apparatus and the haplochromines' mating system are important traits that have contributed to the evolutionary success of cichlid fishes in general and of haplochromines in particular $[7,13,14,38]$. The adaptive advantage of the mating system of the haplochromines (with coloration and egg-spots as sexual advertisement) might be the facilitation of sexual selection through female choice (see e.g., $[7,16,21])$. Sexual selection has been suggested as a major cause for the explosive origin of new species of cichlids in species flocks $[3,13,39,40]$.

\section{csf I ra is also expressed in egg-dummies of ectodine cichlids}

One of the most fascinating aspects of cichlid evolution is the repeated occurrence of evolutionary parallelisms [1,41-43]. This has led to the question of whether natural selection alone is sufficient to produce parallel morphologies or whether a developmental or genetic bias has influenced the direction of diversification [6]. Because of their independent origin in at least two lineages of mouthbrooding cichlids (not counting the genital tassels of some Tilapia species), egg-dummies on cichlid fins are likely to represent another example of evolutionary parallelism in the adaptive radiations of cichlids in East Africa - in this case involving a rather complex ethomorphological trait.

The function of egg-dummies in mimicking eggs to attract females is known from haplochromines and also from ectodines such as O. ventralis [14] (Figure 3v). Nevertheless, the dummies of $O$. ventralis (and its congeners) and those of haplochromines are of independent evolutionary origin, and they show different degrees of complexity. Most importantly, the two kinds of egg-markings are found on different anatomical structures, leading to substantial differences in the spawning behavior. In male haplochromines, the often numerous egg-spots are situated on the anal fin and, hence, are in close proximity to the genital opening to which the female's mouth is supposed to be guided (Figure 1). In O. ventralis (and its congeners) two blotches each are found on the tassels at the tips of the paired pelvic fins, which are conspicuously elongated (Figure 3v, w). Spawning in O. ventralis takes place in huge sand bowers of a diameter of up to half a meter in size, which are built by the territorial males in 
Table I: List of specimens used in this study and taxonomic information. The tribe names follow the nomenclature of [57,58]. Note that the 'modern haplochromines' are a monophyletic subgroup of the haplochromine cichlids and contain the entire species flocks of Lake Malawi and the Victoria region, the Tanganyikan Tropheini, as well as some riverine and lacustrine haplochromines in East Africa [7]. Species that exhibit egg-dummies on male anal fins (AF) or pelvic fins (PF) are indicated.

\begin{tabular}{|c|c|c|c|c|}
\hline \multirow[b]{2}{*}{ Species name } & \multirow[b]{2}{*}{ Geographic origin } & \multirow[b]{2}{*}{ Tribe/taxonomic information } & \multicolumn{2}{|c|}{ Egg-spots } \\
\hline & & & $\mathbf{A F}$ & PF \\
\hline Altolamprologus calvus & Lake Tanganyika & Lamprologini & & \\
\hline Astatoreochromis alluaudi & East Africa, Lake Victoria region & Haplochromini & $\mathrm{X}$ & \\
\hline Astatotilapia burtoni & Lake Tanganyika area & Haplochromini/'modern haplochromines' & $\mathrm{X}$ & \\
\hline Bathybates minor & Lake Tanganyika & Bathybatini & & \\
\hline Cyrtocara moorii & Lake Malawi & Haplochromini/'modern haplochromines' & $*$ & \\
\hline Haplochromis bloyeti & East Africa, rivers & Haplochromini/'modern haplochromines' & $\mathrm{X}$ & \\
\hline Haplochromis obliquidens & Lake Victoria & Haplochromini/'modern haplochromines' & $\mathrm{X}$ & \\
\hline Lamprologus teugelsi & Congo River & Lamprologini & & \\
\hline Limnochromis auritus & Lake Tanganyika & Limnochromini & & \\
\hline Ophthalmotilapia ventralis & Lake Tanganyika & Ectodini & & $\mathrm{X}$ \\
\hline Oreochromis tanganicae & Lake Tanganyika & Tilapiini & & \\
\hline Plecodus straeleni & Lake Tanganyika & Perissodini & & \\
\hline Pseudocrenilabrus multicolor & East Africa, rivers and lakes & Haplochromini & $\mathrm{X}$ & \\
\hline Telmatochromis bifrenatus & Lake Tanganyika & Lamprologini & & \\
\hline Pseudotrophus sp. 'bicolor' & Lake Malawi & Haplochromini/'modern haplochromines' & $\mathrm{X}$ & \\
\hline Thoracochromis brauschi & Congo River & Haplochromini/CSA clade** & $\mathrm{X}$ & \\
\hline Trematocara unimaculatum & Lake Tanganyika & Trematocarini & & \\
\hline Xenotilapia spiloptera & Lake Tanganyika & Ectodini & & \\
\hline Xystichromis phytophagus & Lake Kanyaboli, Lake Victoria & Haplochromini/'modern haplochromines' & $\mathrm{X}$ & \\
\hline
\end{tabular}

*This species from Lake Malawi is a haplochromine representative lacking anal fin egg-spots.

**CSA clade, Congolese/South African clade (according to [7]).

order to attract females. An interested female lays a few eggs in the center of the bower and picks them up into her mouth, after which the male displays its ventral fins with their yellow markings at the egg-laying spot. The female takes up the tassels into her mouth. The tips of the pelvic fins are put into close proximity to the male's genital opening, which discharges sperm [14].

Here, we provide evidence that the same gene is expressed in both kinds of egg-dummies. Just as in the haplochromine species examined, csf1 ra is expressed in the yellow blotches on the tassels at the tips of the paired ventral fins of males of $O$. ventralis, whereas females do not show csf1 ra expression (Figure $3 \mathrm{y}, \mathrm{z}$ ). This observation primarily indicates that both kinds of egg dummies are made up of xanthophores, for which csflra is a good marker gene. The two kinds of independently evolved egg dummies might, in the future, serve as model system to test whether the same genetic pathways are involved in the morphogenesis of a complex ethomorphological trait.

\section{Methods \\ Cichlid fish}

Cichlids for in situ hybridizations and molecular evolutionary analyses were reared in the Tierforschungsanlage at the University of Konstanz, Germany under standard conditions (12 h light/12 h dark; $26^{\circ} \mathrm{C}$ ). Additional cich- lid species were collected in East Africa in autumn 2004. We included representative species of the main haplochromine lineages [7]: Astatotilapia burtoni (Lake Tanganyika and surrounding rivers), Astatoreochromis alluaudi (lakes and rivers of the Lake Victoria region), Cyrtocara moorii (Lake Malawi), Haplochromis bloyeti (East African rivers), Haplochromis obliquidens (Lake Victoria), Pseudotropheus sp. 'bicolor' (Lake Malawi), Pseudocrenilabrus multicolor (East African lakes and rivers; Nile system), Thoracochromis brauschi (Congo drainage), and Xystichromis phytophagus (Lake Kanyaboli; belongs to the Lake Victoria region superflock) (Table 1). The non-haplochromine representatives were: Altolamprologus calvus, Bathybates minor, Limnochromis auritus, Ophthalmotilapia ventralis, Oreochromis tanganicae, Plecodus straeleni, Telmatochromis bifrenatus, Trematocara unimaculatum, and Xenotilapia spiloptera (all belong to the Lake Tanganyika species assemblage), and Lamprologus teugelsi (Congo drainage) (see [44] for taxonomic information, and $[7,8,22]$, and this study for phylogenetic assignments). The animals were anesthetized with MS222 (Sigma, Deisenhofen, Germany) prior to manipulations.

\section{Fluorescence visualization of xanthophores}

For fluorescence visualization of xanthophores in cichlid fins, we used a modified version of the method described in [45]. Amputated anal, dorsal and ventral fins of haplo- 
chromine cichlids were mounted in 5\% methylcellulose $(500 \mu \mathrm{l})$ with dilute ammonia $(25 \mu \mathrm{l})$, and $\beta$-mercaptoethanol, pH $10(1 \mu \mathrm{l})$. Digital images were taken with a Zeiss AxioCam Mrc digital camera using a Zeiss Axioplan2 stereomicroscope (Zeiss, Jena, Germany).

\section{Reverse transcriptase mediated PCR}

Prior to in situ hybridization experiments, we confirmed expression of csf1ra in the haplochromine egg-spots by means of a reverse transcriptase mediated PCR. We amplified and sequenced a 540-bp fragment of csf1ra from cDNA that was transcribed from mRNA extracted from $A$. burtoni egg-spot tissue, using the primers F_1986 5'-GCTGCCCTACAATGAAAAGTG-3' and R_2186 5'-TTGACGATGTTCTGGTGGTGA-3'.

\section{In situ hybridization experiments}

A 1 233-bp fragment of csf1 ra was amplified by PCR from A. burtoni cDNA using primers F_1986 5'-GCTGCCCTACAATGAAAAGTG-3' and R_3199 5'-AYTGRTAGTTRTTGGKCTTCA-3'. The amplified fragment was cloned into the pCRII vector using the TA Cloning Dual Promoter Kit (Invitrogen, Karlsruhe, Germany). The orientation of ligated inserts with respect to Sp6 and T7 promoters was determined by direct sequencing on an ABI 3100 capillary sequencer using the BigDye terminator reaction chemistry (Applied Biosystems, Darmstadt, Germany). For in situ hybridization experiments, DIG-labeled (Roche) antisense RNA was transcribed from a linearized fragment using DIG-labeled dNTPs.

Four rounds of in situ hybridization experiments were performed. In total, five male anal fins (plus two female anal fins) were studied from $P$. sp. 'bicolor' and seven male anal fins (plus one female and one dorsal fin) from A. burtoni. Furthermore, we used six anal fins (plus one dorsal fin) from $P$. multicolor, three male anal fins from $T$. brauschi, and three male plus two female ventral fins from O. ventralis. Amputated fins were fixed in $4 \%$ paraformaldehyde in phosphate buffer saline (PFA/PBS) at $4{ }^{\circ} \mathrm{C}$ overnight, washed twice in PBTw $(0.1 \%$ Tween-20 in PBS, 0.01 DEPC), and stored in 100\% methanol. Then, fins were rehydrated (PBTw washed and post fixed in 4\% PFA/PBS) and treated with proteinase $K$ (Roche) for $10 \mathrm{~min}$ at a final concentration of $14 \mu \mathrm{g} / \mathrm{ml}$ (see [46]). After PBTw washing, cichlid fins were prehybridized (50\% formamide, $5 \times$ SSC, $1 \mathrm{mg} / \mathrm{ml}$ tRNA, $50 \mu \mathrm{g} / \mathrm{ml}$ heparin, $0.1 \%$ Tween-20, $9 \mathrm{mM}$ citric acid, $\mathrm{pH}$ 6.0) and hybridized at $69^{\circ} \mathrm{C}$ overnight in hybridization buffer plus 1/10 volume of labeled probe. Fins were gradually transferred to PBTw and blocking solution (Boehringer Mannheim, Mannheim, Germany). Anti-(DIG-AP) antibody (Roche) in 0.5\% blocking solution and BCIP/X-phos (Roche) were used to visualize target RNA. The tissue was finally fixed in $4 \%$ PFA/PBS and stored in $70 \%$ glycerol/PBS. As negative control, we applied labeled sense RNA to male fins of A. burtoni and $P$. sp. 'bicolor'. Photos of live fins and stained tissue were taken with a Zeiss AxioCam Mrc digital camera using a Zeiss Axioplan2 and Stemi SV11 APO stereomicroscopes. Photos were processed with AxioVision 3.1 (Zeiss) and Photoshop 7.0 (Adobe) software; the background of images was modified with Photoshop 7.0 (Adobe, San Jose, California, USA).

\section{Polymerase chain reaction and DNA sequencing: the csfIra locus}

For PCR amplification of genomic DNA of 18 East African cichlid species, we used the primers listed in Table 2 that had been designed against a reference sequence from $A$. burtoni (GenBank accession number: DQ386648; [24]). We also resequenced the A. burtoni locus with these primers. Exon 1 and the relatively large introns 1, 16, and 19 were not sequenced. The primers for amplification of the upstream region of csf $1 \mathrm{ra}$ were 26M7_3F 5'-CTCACCTCTGCGGATGTTTC-3' and 26M7_3R 5'-GCCACAGCATAAGGAAGGAC-3'. For the determination of the cDNA sequence of csf1ra from a normalized library ("pinky") made from different $A$. burtoni mRNA pools [47], we additionally used the primers listed in Table 3. For comparative analyses, we also determined, for the same set of species, two segments of another type III receptor tyrosine kinase, kita. This pigmentation gene plays an important role in melanophore development [31]. It is therefore another candidate for being an important color gene in cichlid fishes. We determined a ca. $300 \mathrm{bp}$ segment in the

Table 2: Primers used for sequencing of the csflra locus in 19 cichlid specimens

\begin{tabular}{ll}
\hline Primer & Sequence \\
\hline ex2_3F & 5'-AAAACCCTCAGAGACCATCAG-3' \\
ex2_3R & 5'-TCCAATAAACGCATCAGAGAG-3' \\
ex4_6F & 5'-GGTAGAGCAGGTGGTTCAGTC-3' \\
ex4_6R & 5'-CAGCACTCTTCCCTCTTTGAG-3' \\
ex7_9F & 5'-GAAAAGTGCGAAAATCAAGTG-3' \\
ex7_9R & 5'-TCAGAAGGGAAGTAAAGGGC-3' \\
ex10_IIF & 5'-ATTACTGGATGTGGTTCAGATC-3' \\
ex10_IIR & 5'-ACAGAGGAGCATTTTGACTTC-3' \\
ex13_16F & 5'-TTTTTGAGTTGAGCGTGACAG-3' \\
ex13_I6R & 5'-AACCTCACACCCATCCTGC-3' \\
exI7_I9F & 5'-TCTTATTGTCTTTCACTGGGC-3' \\
exI7_I9R & 5'-ATCAGTCGTAAAAACTCTGCTG-3' \\
F_1986 & 5'-GCTGCCCTACAATGAAAAGTG-3' \\
R_2186 & 5'-TTGACGATGTTCTGGTGGTGA-3' \\
F_296I & 5'-ARATGTGCTGGAAYCTGGA-3' \\
R_3199 & 5'-AYTGRTAGTTRTTGGKCTTCA-3' \\
F_1962 & 5'-ACTACACCTTYRTYGACCCCAC-3' \\
R_2269 & 5'-CARRAAGTTSAGCAGGTCGCC-3' \\
F_1758 & 5'-CCCACATCACAAAGCACAGA-3' \\
R_2425 & 5'-GCCAGTGGGAAGAAATGAG-3' \\
F_0732 & 5'-TAAGGCCTTTTCCATCAATG-3' \\
R_14I7 & 5'-GCGATAGTCAGTGTGCTCTC-3' \\
\end{tabular}


Table 3: Primers used for cDNA amplification and sequencing

\begin{tabular}{ll}
\hline Primer & Sequence \\
\hline I0F_exI & 5'-TACCTTATGCTGTGGCTTGTG-3' \\
I557F_ex2 & 5'-GATCTGAGGTGTGAGGGTGAA-3' \\
4I34F_ex9 & 5'-GAGTACGGGGCTGTGGAAGTG-3' \\
73I5F_exI7 & 5'-AGAGATGTGGCTGCGAGGATT-3' \\
3650R_ex7 & 5'-TGCGTTGGTCATTTCACTCTG-3' \\
6030R_ex16 & 5'-CTTGGAAGGAAAATCTGAGCA-3'
\end{tabular}

extracellular domain of kita (including the second immunoglobulin domain) using primers Burt-Kit-F-474 5'GATCTGGAGAATATGCACCTGGA-3' and Burt-Kit-R-672 5'-ATCACTCTTGTGGATGGTTGGAG-3' and a ca. 500 bp segment corresponding to the kinase insert domain using primers a Burt-Kit-F-2023 5'-TATTGTCAACCTACTGGGAGC C-3' and Burt-Kit-R-2316 5'-AACCGTCATCAGCAAACATCTC-3'. PCR reactions were carried out under standard conditions on ABI 9700 thermocyclers (Applied Biosystems). PCR products were purified with Qiaquick spin columns (Qiagen, Hilden, Germany). Sequencing reactions were performed with forward and reverse primers (see above for details) using the BigDye terminator reaction chemistry (Applied Biosystems). DNA sequences were detected on ABI 3100 automated capillary DNA sequencers (Applied Biosystems).

\section{Molecular evolutionary analyses: the csf I ra locus}

DNA sequences were quality trimmed with Phred [48] and assembled with Sequencher 3.0 [49]. Sequences have been deposited in GenBank under the accession numbers EU042675-EU042749 (csf1ra) and EU042637EU042674 (kita) (note that the segment spanning exons 17 to 19 in csf1ra could not be amplified for T. unimaulatum). Exon/intron boundaries were identified using a reference sequence from A. burtoni (DQ386648) and checked by homology comparison with reference

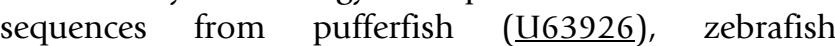
(AF240639) and trout (AJ417832). The protein structure of Csf1ra and the functional domains were identified from homologous sequence motifs in zebrafish (using the Protein families database Pfam [50]) and pufferfish [23]. MatInspector from Genomatix [51] was used to identify putative promoter modules in the upstream regions of csfira.

Maximum likelihood and maximum parsimony phylogenetic analyses with 19 cichlid taxa were performed with Paup * 4.0b10 [52]. The appropriate model parameters for the maximum likelihood analysis were determined by means of a likelihood ratio test with Modeltest version 3.6 [53]. For the maximum likelihood tree search based on the non-coding section of the csf1ra locus (4 $171 \mathrm{bp}$ ), we used the general time reversible model of molecular evo- lution (six types of substitutions) with a proportion of invariable sites of 0.4714 and a gamma substitution correction $(\alpha=0.8669)$. An unweighted heuristic maximum parsimony search was performed with the same dataset (50 replicates). Bootstrap analyses were performed with 100 replicates under the maximum likelihood criterion and with 1000 replicates for maximum parsimony analysis. Alternative branching orders at critical branches within the haplochromines were evaluated by means of a nonparametric Shimodaira-Hasegawa test under a resampling-estimated log likelihood with 1000 bootstrap replicates as implemented in Paup*: The optimal maximum likelihood topology with Pseudocrenilabrus multicolor as sistergroup to Thoracochromis brauschi and all remaining haplochromines was tested against trees in which $T$. brauschi or Astatoreochromis alluaudi were forced to occupy the most ancestral position within the haplochromines (see also [7]).

Sliding window analyses for calculating the nucleotide diversity $(\pi)$ in the coding region of csf1ra in haplochromines versus non-haplochromines were performed with DNASP 4.0 [30]. We used a window size of $50 \mathrm{bp}$ and an overlap of $10 \mathrm{bp}$. We also used DNASP for the calculation of the $d \mathrm{~N} / d \mathrm{~S}$ ratio in haplochromines compared to non-haplochromines using the same 50-bp windows (overlap: $10 \mathrm{bp}$ ). We then used HyPhy [54] for the reconstruction of $d \mathrm{~N} / d \mathrm{~S}$ ratios, based on maximum likelihood, on the branches of the phylogeny obtained before (see above), analyzing the entire dataset as well as the first 1 551 bp only, which correspond to the extracellular ligandbinding domain of csf1 ra. We applied a site-to-site variation model with two independent gamma distributions and the MG94 model, and tested for relevant internal branches in the tree. The reconstructed $d \mathrm{~N} / d \mathrm{~S}$ ratios were visualized in form of a branch-scaled tree applying the Suzuki-Gojobori derived adaptive selection tool implemented in HyPhy. We also used DNASP for sliding window analyses in kita and HyPhy to plot $d \mathrm{~N} / d \mathrm{~S}$ ratios for the two segments of kita on the maximum likelihood tree.

\section{Character state reconstructions}

The characteristic egg-dummies of the haplochromines have been identified as a potential key evolutionary innovation that might be directly related to the evolutionary success of this most species-rich group of cichlid fishes [7] (but see [55]). As some ancestral (but also some derived) species of haplochromines do not show egg-dummies on male anal fins, we intended to reconstruct the evolutionary origin of these markings based on the new phylogenetic and comparative morphological data now available. Specifically, we wanted to evaluate the hypothesis that these characteristic egg-spots evolved only once in the ancestor of the haplochromines and that the missing ovoid markings on anal fins of males of some species are 
due to secondary loss. We used Mesquite 1.03 [56] for maximum likelihood and maximum parsimony ancestral state reconstructions of the evolutionary origin of eggdummies on male anal fins, on the basis of a consensus phylogeny of East African cichlid fishes. We also mapped the evolutionary origin of the characteristic polygynous mating system with maternal mouthbrooding involving egg-spots on that phylogeny. The consensus tree was built using a previous mitochondrial phylogeny [7] as well as the present phylogenetic hypothesis that is based on nuclear DNA. Note that the ancestral polytomy between the Pseudocrenilabrus, the Astatoreochromis, the Congolese/ South African lineage, and the modern haplochromines [7] remained unresolved in all available phylogenetic hypotheses.

\section{Authors' contributions}

WS, IB and AM designed the study. WS and IB carried out the molecular work and the analyses. All authors contributed to the preparation of the manuscript, and read and approved the final version.

\section{Additional material}

\section{Additional file 1}

The breeding cycle of Astatotilapia burtoni. Modified from [59]. Click here for file

[http://www.biomedcentral.com/content/supplementary/17417007-5-51-S1.mov]

\section{Additional file 2}

Amino acid substitutions in Csf1 ra in haplochromines. The numbering of amino acids is relative to the start site in $\mathrm{A}$. burtoni (DQ386648). Click here for file

[http://www.biomedcentral.com/content/supplementary/1741-

7007-5-51-S2.jpeg]

\section{Additional file 3}

$\mathrm{dN} / \mathrm{d} S$ ratio of haplochromines compared to non-haplochromines in two segments of kita. The sliding window analysis with DNASP did not detect a $d N / d S>1$ in the extracellular domain (a) or in the intracellular domain (b) of kita. See Figure $4 b$ for the same analysis in csf1ra and the Methods section for details of the analysis.

Click here for file

[http://www.biomedcentral.com/content/supplementary/17417007-5-51-S3.jpeg]

\section{Acknowledgements}

We thank $C$ Chang-Rudolf, $Y$ Gibert, N Offen, $N$ Sigel and T Wirth for technical assistance; $M$ Barluenga, G Begemann, P Bunje, B Egger, H Hoekstra, $\mathrm{H}$ Hofmann, M Nachmann, D Parichy, T Wirth and two anonymous reviewers for discussion and valuable suggestions on the manuscript. WS was supported by a Marie Curie Fellowship of the EU, and grants from the Landesstiftung-Baden Württemberg gGmbH and the Center for Junior Research Fellows, University of Konstanz; AM was supported by the Deutsche Forschungsgemeinschaft (DFG) and the University of Konstanz.

\section{References}

I. Stiassny MLJ, Meyer A: Cichlids of the Rift lakes. Sci Am 1999 , 280:64-69.

2. Salzburger W, Meyer A: The species flocks of East African cichlid fishes: recent advances in molecular phylogenetics and population genetics. Naturwissenschaften 2004, 9 1:277-290.

3. Kocher TD: Adaptive evolution and explosive speciation: the cichlid fish model. Nat Rev Genet 2004, 5:288-298.

4. Schluter D: The Ecology of Adaptive Radiation Oxford, UK: Oxford University Press; 2000.

5. Albertson RC, Kocher TD: Genetic and developmental basis of cichlid trophic diversity. Heredity 2006, 97:2III-22I.

6. Brakefield PM: Evo-devo and constraints of selection. Trends Ecol Evol 2006, 21:362-368.

7. Salzburger W, Mack T, Verheyen E, Meyer A: Out of Tanganyika: genesis, explosive speciation, key-innovations and phylogeography of the haplochromine cichlid fishes. BMC Evol Biol 2005, 5: 17 .

8. Verheyen E, Salzburger W, Snoeks J, Meyer A: Origin of the superflock of cichlid fishes from Lake Victoria, East Africa. Science 2003, 300:325-329.

9. Turner GF, Seehausen O, Knight ME, Allender C], Robinson RL: How many species of cichlid fishes are there in African lakes? Mol Ecol 200I, 10:793-806.

10. Greenwood PH: Towards a phyletic classification of the 'genus' Haplochromis (Pisces, Cichlidae) and related taxa. Bull Br Mus nat Hist (Zool) 1979, 35:265-322.

II. Wickler W: 'Egg-dummies' as natural releasers in mouthbreeding cichlids. Nature 1962, 194:1092-1093.

12. Wickler W: Zur Stammesgeschichte funktionell korrelierter Organ- und Verhaltensmerkmale: Ei-Attrappen und Maulbrüten bei afrikanischen Cichliden. Zeitschrift für Tierpsychologie 1962, 19:129-164.

13. van Alphen JJ, Seehausen O, Galis F: Speciation and radiation in African haplochromine cichlids. In Adaptive Speciation Edited by: Dieckmann U, Doebeli M, Metz JAJ, Tautz D. Cambridge, UK: Cambridge University Press; 2004.

14. Fryer G, lles TD: The Cichlid Fishes of the Great Lakes of Africa: Their Biology and Evolution Edinburgh: Oliver \& Boyd; 1972.

15. Kirchshofer R: Aktionssystem des Maulbrüters Haplochromis desfontainesii. Zeitschrift für Tierpsychologie 1953, 10:297-318.

16. Goldschmidt T: Egg mimics in haplochromine cichlids (Pisces, Perciformes) from Lake Victoria. Ethology 1991, 88:177-190.

17. Hert $E$ : The function of egg-spots in an African mouth-brooding cichlid fish. Anim Behav 1989, 37:726-732.

18. Mrowka W: Egg stealing in a mouthbrooding cichlid fish. Anim Behav 1987, 35:923-925.

19. Konings A: Malawi Cichlids in their Natural Habitat 3rd edition. El Paso, TX: Cichlid Press; 200I.

20. Couldridge VCK: Experimental manipulation of male eggspots demonstrates female preference for on large spot in Pseudotropheus lomardoi. J Fish Biol 2002, 60:726-730.

21. Goldschmidt T, de Visser J: On the possible role of egg mimics in speciation. Acta Biotheoretica 1990, 38: I25-134.

22. Salzburger W, Meyer A, Baric S, Verheyen E, Sturmbauer C: Phylogeny of the Lake Tanganyika cichlid species flock and its relationship to the Central and East African haplochromine cichlid fish faunas. Syst Biol 2002, 5 I: I I3-135.

23. Rousset D, Agnes F, Lachaume P, Andre C, Galibert F: Molecular evolution of the genes encoding receptor tyrosine kinase with immunoglobulin-like domains. J Mol Evol 1995, 41:42I-429.

24. Braasch I, Salzburger W, Meyer A: Asymmetric evolution in two fish-specifically duplicated receptor tyrosine kinase paralogons involved in teleost coloration. Mol Biol Evol 2006, 23:1192-1202.

25. Parichy DM, Ransom DG, Paw B, Zon LI, Johnson SL: An orthologue of the kit-related gene fms is required for development of neural crest-derived xanthophores and a subpopulation of adult melanocytes in the zebrafish, Danio rerio. Development 2000, I 27:303I-3044.

26. Parichy DM, Turner JM: Temporal and cellular requirements for Fms signaling during zebrafish adult pigment pattern development. Development 2003, 130:817-833.

27. Quigley IK, Manuel JL, Roberts RA, Nuckels RJ, Herrington ER, MacDonald EL, Parichy DM: Evolutionary diversification of pigment 
pattern in Danio fishes: differential fms dependence and stripe loss in D. albolineatus. Development 2005, 132:89- 104.

28. Clabaut C, Salzburger W, Meyer A: Comparative phylogenetic analyses of the adaptive radiation of Lake Tanganyika cichlid fish: nuclear sequences are less homoplasious but also less informative than mitochondrial DNA. J Mol Evol 2005, 6I:666-68I.

29. Wiesmann C, Muller YA, de Vos AM: Ligand-binding sites in Iglike domains of receptor tyrosine kinases. I Mol Med 2000 78:247-260.

30. Rozas J, Sanchez-DelBarrio JC, Messeguer X, Rozas R: DnaSP: DNA polymorphism analyses by the coalescent and other methods. Bioinformatics 2003, 19:2496-2497.

31. Parichy DM, Rawls JF, Pratt SJ, Whitfield TT, Johnson SL: Zebrafish sparse corresponds to an orthologue of c-kit and is required for the morphogenesis of a subpopulation of melanocytes, but is not essential for hematopoiesis or primordial germ cell development. Development 1999, I 26:3425-3436.

32. Schlessinger J: Cell signaling by receptor tyrosine kinases. Cell 2000, I03:2।I-225.

33. Protas ME, Hersey C, Kochanek D, Zhou Y, Wilkens H, Jeffery WR, Zon LI, Borowsky R, Tabin CJ: Genetic analysis of cavefish reveals molecular convergence in the evolution of albinism. Nat Genet 2006, 38: 107-III.

34. Terai $Y$, Morikawa N, Okada N: The evolution of the pro-domain of bone morphogenetic protein 4 (Bmp4) in an explosively speciated lineage of East African cichlid fishes. Mol Biol Evol 2002, 19:1628-1632.

35. Albertson RC, Streelman JT, Kocher TD, Yelick PC: Integration and evolution of the cichlid mandible: the molecular basis of alternate feeding strategies. Proc Natl Acad Sci USA 2005, 102: 16287-16292.

36. Terai Y, Mayer WE, Klein J, Tichy H, Okada N: The effect of selection on a long wavelength-sensitive (LWS) opsin gene of Lake Victoria cichlid fishes. Proc Natl Acad Sci USA 2002, 99:1550I-15506.

37. Terai Y, Morikawa N, Kawakami K, Okada N: Accelerated evolution of the surface amino acids in the WD-repeat domain encoded by the hagoromo gene in an explosively speciated lineage of East African cichlid fishes. Mol Biol Evol 2002 , 19:574-578.

38. Liem KF: Evolutionary strategies and morphological innovations: cichlid pharyngeal jaws. Syst Zool 1973, 22:425-44I.

39. Danley PD, Kocher TD: Speciation in rapidly diverging systems: lessons from Lake Malawi. Mol Ecol 200I, I0:1075-1086.

40. Turner GF, Burrows MT: A model of sympatric speciation by sexual selection. Proc $R$ Soc Lond B Biol Sci 1995, 260:287-292.

4I. Kocher TD, Conroy JA, McKaye KR, Stauffer JR: Similar morphologies of cichlid fish in lakes Tanganyika and Malawi are due to convergence. Mol Phylogenet Evol 1993, 2:158-165.

42. Rüber L, Verheyen E, Meyer A: Replicated evolution of trophic specializations in an endemic cichlid fish lineage from Lake Tanganyika. Proc Natl Acad Sci USA 1999, 96: 10230-10235.

43. Sturmbauer C, Hainz U, Baric S, Verheyen E, Salzburger W: Evolution of the tribe Tropheini from Lake Tanganyika: synchronized explosive speciation producing multiple evolutionary parallelism. Hydrobiologia 2003, 500:5I-64.

44. Fishbase [http://www.fishbase.org]

45. Odenthal J, Rossnagel K, Haffter P, Kelsh RN, Vogelsang E, Brand M, van Eeden FJ, Furutani-Seiki M, Granato M, Hammerschmidt M, et al: Mutations affecting xanthophore pigmentation in the zebrafish, Danio rerio. Development 1996, I 23:391-398.

46. Zauner H, Begemann G, Mari-Beffa M, Meyer A: Differential regulation of msx genes in the development of the gonopodium, an intromittent organ, and of the "sword," a sexually selected trait of swordtail fishes (Xiphophorus). Evol Dev 2003, 5:466-477.

47. Salzburger W, Renn SC, Steinke D, Braasch I, Hofmann H, Meyer A: Annotation of expressed sequence tags for the East African cichlid fish Astatotilapia burtoni and evolutionary analyses of cichlid ORFs.

48. Phred [http://www.phrap.com]

49. Sequencher 3.0 [http://www.genecodes.com/]

50. Pfam [http://www.sanger.ac.uk/cgi-bin/Pfam/]

51. Matlnspector [http://www.genomatix.de/]
52. Swofford DL: PAUP* - Phylogenetic Analyses Using Parsinomy and other methods, version 4.0 Sunderland, MA: Sinauer; 2003.

53. Posada $D$, Crandall KA: MODELTEST: testing the model of DNA substitution. Bioinformatics 1998, |4:8|7-8|8.

54. Pond SL, Frost SD, Muse SV: HyPhy: hypothesis testing using phylogenies. Bioinformatics 2005, 21 :676-679.

55. Seehausen O: African cichlid fish: a model system in adaptive radiation research. Proc Biol Sci 2006, 273:1987-1998.

56. Maddison WP, Maddison DR: Mesquite: A modular system for evolutionary analysis. [http://mesquiteproject.org].

57. Poll M: Classification des Cichlidae du lac Tanganika, Tribus, genres et espéces 2nd edition. Brussels, Belguim: Académie Royale de Belgique; 1986.

58. Takahashi T: Systematics of Tanganyikan cichlid fishes (Teleostei: Perciformes). Ichthyol Res 2003, 50:367-382.

59. Wickler W: Haplochromis burtoni (Cichlidae) Ablaichen. In Encyclopedia Cinematographica E470 Institut für den wissenschafltichen Film, Göttingen, Germany; 1963.

Publish with BioMed Central and every scientist can read your work free of charge

"BioMed Central will be the most significant development for disseminating the results of biomedical research in our lifetime. "

Sir Paul Nurse, Cancer Research UK

Your research papers will be:

- available free of charge to the entire biomedical community

- peer reviewed and published immediately upon acceptance

- cited in PubMed and archived on PubMed Central

- yours - you keep the copyright
BiolMedcentral 